\title{
Selfie syndrome: an infectious gift of IT to health
}

\section{care}

\section{Editorial}

Oxford Dictionary named 'selfie' as its word of the year - an award given to a word or expression that has attracted a significant amount of interest in the past year. According to language research, the use of 'selfie' increased by a colossal 17,000\% between 2012 and 2013, which marks a huge upward trend. Recently new rapidly emerging Information technology syndrome engulfing the young population like a pandemic has been identified, I have no wonder if I coin this syndromes E-selfing syndrome. Its incidence and prevalence is dramatically on rise in the world among all economic class, educated, young society especially in developed world.

\section{Epidemiology}

Exact prevalence is not known but as per the experts, approximately $40 \%$ or higher percentage of teenage, young and adult population in the developed world and somewhat lower in the developing world are suffering from the e-selfing syndrome with the manifestations varying from mild to severe. World wide availability of smart phones, WiFi and mobile data package has worsened this scenario further. One recent survey of Media usage in the USA revealed that the three medias gaining people's time are television as well as desktop and mobile internet. Between 2009 and 2012, average daily TV consumption per capita grew 11 minutes, desktop internet grew 27 minutes, and mobile internet grew by 60 minutes. In 2010, mobile surpassed print media for the first time. On the global level, desktop internet was the most used medium (approximately 260minutes) per capita daily. Television ranked second with 150minutes whereas mobile internet was used for 77 minutes per capita daily on average.

\section{Pathogenesis}

Selfie addiction is a new pathology, often related to past bullying and low self-esteem. Psychiatrists are beginning to consider the compulsion to take selfies as a serious mental health problem, although precise mechanism of e-selfing syndrome is not known but it is assumed to be due to imbalance between the excitatory and inhibitory Neuro transmitter. Serotonergic pathway could also be involved. However this needs further elucidation for biochemical and neuro physiological testing's. A recent study done in USA demonstrated a significant connection between time spent on Face book and negative body image that individuals who spent more time on social media were more likely to compare themselves physically to their friends, which in turn was shown to generate negative attitudes towards their own appearance.

\section{Clinical features}

E-selfing syndrome reflects a combination of the wide spectrum of varying degrees degree of Neuro-psychiatric disorders. Some persons may have underlying stress disorder, self-obsessed and narcissistic generation, obsessive compulsive behaviour, anxiety, mania or even bipolar disorder but a new study suggests that it can even be linked to body dysmorphia. Taking Selfies could lead to narcissism, addiction,
Volume 2 Issue 4 - 2015

\author{
Mayank Vats \\ Rashid Hospital, United Arab Emirates
}

Correspondence: Mayank Vats, Pulmonologist, Intensivist \& Sleep Physician, Rashid Hospital \& Dubai Hospital, Dubai Health Authority, DUBAI, UAE, Tel 97I-55-8825307, 97I-558825306,97104-2209356, Email drmayankvats@gmail.com, drmayankvats@yahoo.co.in

Received: April II, 2015 | Published: April 13, 2015

mental illness and even suicide? This syndrome is characterized by most of the time sticking on the social media network viz face book, Twitter, Instagram, what's app, etc answering or replying unwanted or unrelated questions/concerns, giving comments and liking on all materials available on such site for example any photo, status change, profile change, New car, new dress, new house and so on. Sufferers are checking their social media very frequently even every five to ten seconds to see the updates to check for new mails/posts, new comments by their peers and also replying back by giving comments/ likes and share the posts. When severely affected patients takes their photos of day to day activities and tries to share with all of their friends and also invites then to become part of this social media. Initially starting with fun and time-pass gradually it becomes habit and then addiction. The main motive behind this seems to be exposure of the self-esteem and to show off (pseudo show- off) among the society that they are doing something great which release their stress and depression and makes them feel good. Youth is becoming technologyaddicted and selfie-obsessed and is currently undergoing therapy for OCD and Body Dysmorphic Disorder (an excessive anxiety about personal appearance). The Daily Mail reported on a British teenager who became so obsessed with taking the perfect self portrait that he eventually tried to commit suicide and then treated for technology addiction, OCD and body dysmorphia, and is thought to be the UK's first selfie addict.

\section{Direct consequences}

A recent survey conducted by the American Academy of Facial Plastic and Reconstructive Surgery revealed that selfies are behind a boom in young people seeking plastic surgery contributed to a $10 \%$ increase in nose jobs, $7 \%$ increase in hair transplants and a $6 \%$ increase in eyelid surgery in 2013 compared with 2012.The survey also found that the pervasiveness of social media is driving down the age of people having plastic surgery, with $58 \%$ of the surveyed members reporting an increase in cosmetic surgery and 'injectables' in patients under $30 \mathrm{yrs}$ age. It's hardly a surprise that social media is increasing body dissatisfaction. In 2011, study of 248 girls aged 12-19 at the University of Haifa found that girls who spent more time on Face book were more likely to suffer from a range of conditions, 
including bulimia, anorexia, negative physical self-image and have more of an urge to be on a weight-loss diet.

\section{Complications}

These patients gets easily distracted from their important official work with less productivity and poor performance in the work place, job dissatisfaction, unseen stress, peer pressure (without knowing the root cause), unhealthy family relations, marital conflicts etc. It may lead to orthopaedics complications viz. computer related work injuries, cervical spondylitis, low back pain, fibromyalgia, chronic fatigue syndrome, carpel tunnel, tennis elbow, frozen shoulder etc due to prolonged use of the thumbs for typing, awkward posture of the body while using the phone or computer for social media and even catastrophic road traffic accidents while using phones during driving.

\section{Treatment}

Behavioural counselling emphasizing on self-motivation and selfcontrol on unnecessary use of social media, giving quality time to family and friends. Cognitive behaviour therapy also works wonder.

\section{Conclusion}

If selfies, specifically, are proven in the future to cause these negative mental health issues, it would most likely come as no surprise to experts in the fields of psychology and medicine. Prospective large surveys are needed including a large numbers of persons who are actively involved on such social media to find out the underlying psycho-social factors responsible for E-selfing syndrome and the possible consequences of this syndrome.

\section{Message}

Internet and latest IT technique has revolutionized our life style and made life fast and easy for communication purpose but it's double edged sword so must be used for productive purpose rather than E-selfing.

\section{Acknowledgements \\ None.}

\section{Conflict of interest}

The author declares no conflict of interest. 